\title{
Spitäler sind anders
}

Richard O. Binswanger

* Auftakt einer kleinen Reihe von Kurztexten für Kaderund andere Ärzte mit knappem Zeitbudget.

\section{** Aus stilistischen Gründen wird die männliche Form verwendet. Ärztinnen sind ausdrücklich mitgemeint.}

Korrespondenz:

Dr. med. Richard O. Binswanger Radiologie und Nuklearmedizin FMH

Führungsschule Bodensee

Münsterlingen

Oberer Seeweg 9

CH-8597 Landschlacht

r.binswanger@bluewin.ch

\section{Anders als andere Unternehmen}

Es wird immer wieder gefordert, Spitäler wie Unternehmen aus der übrigen Wirtschaft zu führen bzw. zu «managen». Dann soll alles gut kommen. Unzählige Anstrengungen werden dazu unternommen, Strukturen verändert, ein CEO engagiert, Berater angestellt. Anderswo erfolgreiche Managementmethoden werden eingeführt, das Rechnungswesen umgestellt und vieles mehr. Echte Fortschritte sind selten, viele Versuche sind gescheitert. Warum? Weil Spitäler anders sind als andere Unternehmen. Drei Faktoren unter vielen werden im Folgenden angeführt.

\section{Das doppelte Unternehmensziel}

Oberstes Ziel ist der Nutzen für die Gesundheit der Patienten. Dies allein erfordert grosse Anstrengungen: Infrastruktur, Wissen und Können, Einsatzbereitschaft, Teamarbeit und vieles mehr müssen bereitgestellt und gepflegt werden. Durchschnittlichkeit genügt nicht. Das zweite, überlebenswichtige Ziel ist der wirtschaftliche Erfolg. Ohne diesen kann kein medizinischer Nutzen geschaffen werden [1]. Diese beiden Ziele und deren Exponenten, Ärzte ${ }^{* *}$, Pflegende und medizintechnisches Personal auf der einen, das Management auf der andern Seite, widersprechen sich fundamental. Damit müssen Spitäler leben. Nur der verständnisvolle Dialog zwischen Medizin und ökonomischer Leitung führt zum Erfolg [2, 3].

\section{Ungewissheit}

Tuckermann und Rüegg-Stürm [4] bezeichnen die Bewältigung von Ungewissheit als die zentrale Wertschöpfung im Spital. «Im Arbeitsalltag muss man hierzu unabdingbar «auf Zuruf spontan sselbst organisieren können, um die spezifischen Herausforderungen jeweiliger Behandlungsinteraktionen zu meistern. Wir nennen die damit verbundene Form des Organisierens, das die Behandlungsinteraktion ins Zentrum stellt, interaktionales Organisieren. Interaktionales Organisieren ermöglicht es, eine hochanspruchsvolle und von Überraschungen begleitete Wertschöpfung zu erbringen.» Solche Herausforderungen finden sich in kaum einem anderen Unternehmen.

\section{Profession}

Die Organisation von Spitälern folgt den für die Spezialisierung notwendigen Professionen. Kliniken fassen einzelne Professionen zusammen und sind Unternehmen im Unternehmen. An deren Spitze stehen Per-

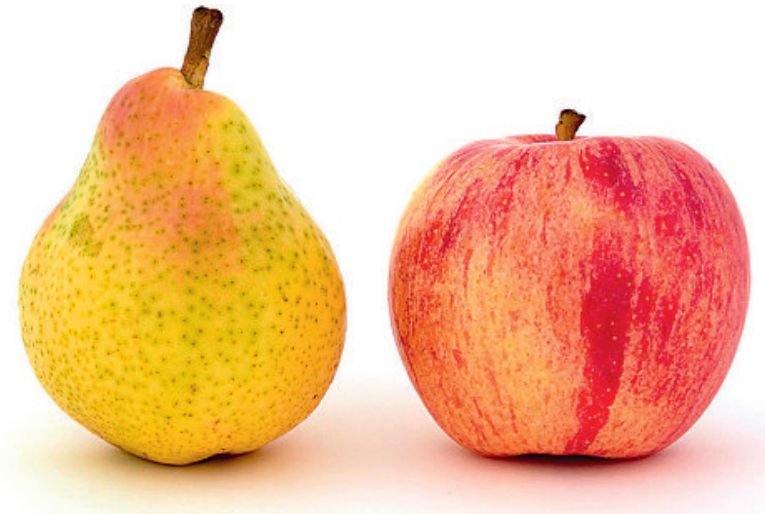

Wer Spitäler mit Unternehmen der übrigen Wirtschaft gleichsetzt, vergleicht Äpfel mit Birnen.

sönlichkeiten von hohem intellektuellem Gewicht. Ein Spital ist differenzierter aufgebaut und in mehr Untereinheiten unterteilt als andere Betriebe. Die Kliniken beanspruchen einen hohen Grad an professioneller Autonomie, das müssen sie tun. Sie stehen aber auch in der Pflicht zur Kooperation - mit den anderen Kliniken und mit dem Management.

\section{Den Unterschied akzeptieren und nutzen}

Nur wer weiss und akzeptiert, dass und wie sich Spitäler von anderen Unternehmen unterscheiden, kann die Aufgabe der Klinik- und Spitalführung optimal bewältigen. Dabei sind Verständnis für die ökonomischen Vorgaben und Zwänge, der Umgang mit Ungewissheit sowie die Kooperation mit allen Beteiligten notwendig und hilfreich.

\section{Literatur}

1 Schultz FC. Who should lead a Healthcare Organization: MDs or MBAs? Journal of Healthcare Management. 2004;49(2):103-116.

2 Glouberman S, Mintzberg H. Managing the Care of Health and the Cure of Disease - Part 1: Differentiation Health Care Management Review.2001;26:55-69

3 Glouberman S, Mintzberg H. Managing the Care of Health and the Cure of Disease - Part 2: Integration Health Care Management Review. 2001;26:70-84

4 Tuckermann H, Rüegg-Stürm J. Sind Spitäler wandelresistent? Organisieren als Stabilisierung von arbeitsteiliger Wertschöpfung im Kontext fundamentaler Ungewissheit. Schweizerische Ärztezeitung. 2007;88(49):1845-1899 